\section{Placement of a novel fully covered metallic stent for refractory pancreatic duct stricture}
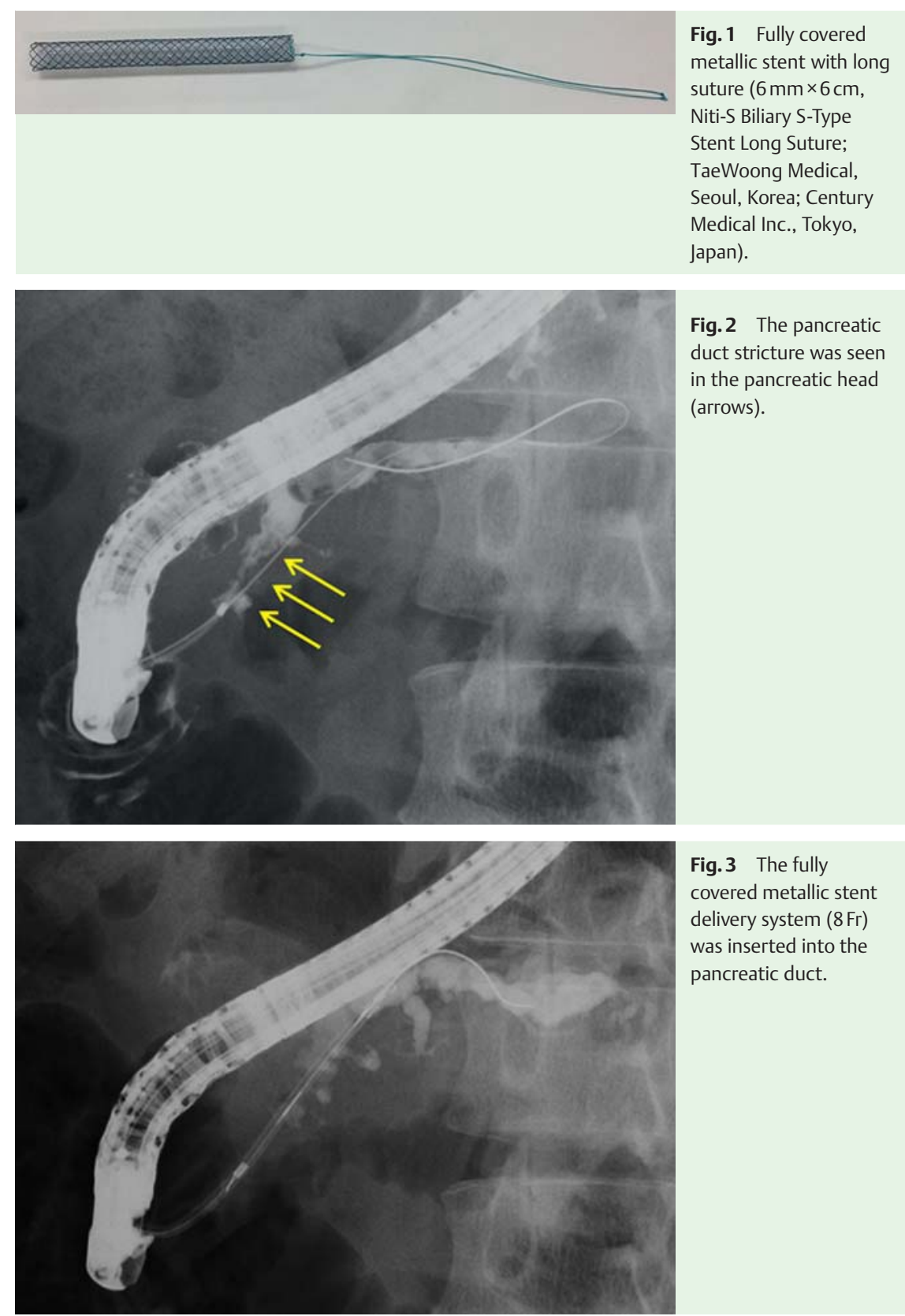

Fig. 3 The fully

covered metallic stent delivery system $(8 \mathrm{Fr})$ was inserted into the pancreatic duct.

Fig. 2 The pancreatic duct stricture was seen in the pancreatic head (arrows).

$$
\text { pancreatic duct. }
$$

Plastic stent placement under endoscopic retrograde cholangiopancreatography is performed widely for the treatment of benign pancreatic stricture [1]. However, this technique may have several disadvantages such as short stent patency and persistent stricture compared with fully covered metallic stents (FCMS) [2,3]. Therefore, although FCMS placement for benign pancreatic stricture is still controversial as a result of a relatively high rate of stent migration (especially distal migration), the FCMS has a potential clinical impact in patients with symptomatic chronic pancreatitis. This report describes the placement of a novel FCMS $(6 \mathrm{~mm} \times 6 \mathrm{~cm}$, Niti-S Biliary S-Type Stent Long Suture; TaeWoong Medical, Seoul, Korea; Century
Medical Inc., Tokyo, Japan) in patients with chronic pancreatitis who have refractory pancreatic duct stricture ( $\bullet$ Fig. 1 ). This novel FCMS has a long removal suture, and therefore, if distal stent migration does occur, the stent can be removed easily by grasping the suture thread.

A 66-year-old man was admitted to the Osaka Medical College Hospital with abdominal pain caused by pancreatic stent occlusion. He had undergone placement of a 10-Fr plastic stent 1 month earlier for pancreatic duct stricture caused by chronic pancreatitis. Despite several stent exchanges, the pancreatic duct stricture persisted. Therefore, it was decided to place a novel FCMS.

First, the plastic stent was removed, and contrast medium was injected into the main pancreatic duct. A pancreatic duct stricture of the pancreatic head was seen ( $\bullet$ Fig.2). Next, the novel metallic stent delivery system (8Fr) was inserted through the stenosis site ( $\bullet$ Fig.3), and the stent was successfully placed ( $\bullet$ Fig. 4 - Video 1). No adverse events occurred and the patient was discharged. After 6 months, no late adverse events, including stent migration, were observed.

This stent may be a safer device because if the stent migrates it can be retrieved easily by grasping the long suture thread. However, results from a prospective study with long term follow-up are needed to confirm this.

\section{Video 1}

The contrast medium was injected into the pancreatic duct, and a stricture was seen in the pancreatic head. A fully covered metallic stent delivery system was inserted, and the stent was successfully placed.

Endoscopy_UCTN_Code_TTT_1AR_2AZ

Competing interests: None

\section{Takeshi Ogura', Yoshitaka Kurisu², Kazuhiro Yamamoto ${ }^{3}$, Daisuke Masuda', Akira Imoto', Shinya Fukunishi ${ }^{1}$, Kazuhide Higuchi ${ }^{1}$}

1 2nd Department of Internal Medicine, Osaka Medical College, Osaka, Japan

2 Department of Pathology, Osaka Medical College, Osaka, Japan

${ }^{3}$ Department of Radiology, Osaka Medical College, Osaka, Japan 


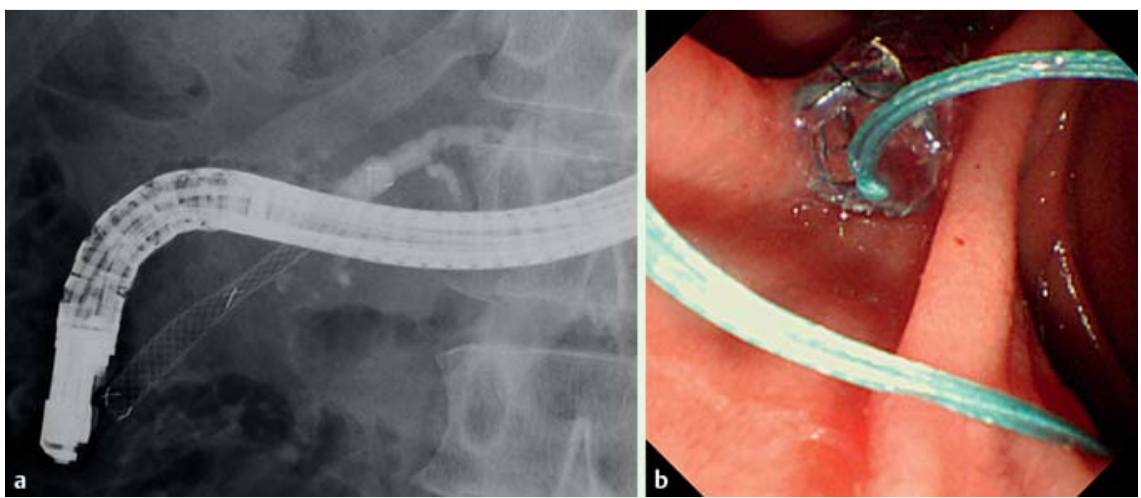

Fig. 4 The stent was successfully placed from the pancreatic body to the head across the stricture site. a Fluoroscopic image. b Endoscopic image.

\section{References}

1 Adler DG, Lichtenstein D, Baron TH et al. The role of endoscopy in patients with chronic pancreatitis. Gastrointest Endosc 2006; 63: 933-937

2 Park do H, Kim MH, Moon SH et al. Feasibility and safety of placement of a newly designed, fully covered self-expandable metal stent for refractory benign pancreatic strictures: a pilot study (with video). Gastrointest Endosc 2008; 68: 1182 - 1189

3 Moon SH, Kim MH, Park do $\mathrm{H}$ et al. Modified fully covered self-expandable metal stents with antimigration features for benign pancreatic duct strictures in advanced chronic pancreatitis, with a focus on the safety profile and reducing migration. Gastrointest Endosc 2010; 72: 86-91

\section{Bibliography}

DOI http://dx.doi.org/

10.1055/s-0034-1391652

Endoscopy 2015; 47: E206-E207

(c) Georg Thieme Verlag KG

Stuttgart · New York

ISSN 0013-726X

\section{Corresponding author}

\section{Takeshi Ogura, PhD, MD}

2nd Department of Internal Medicine

Osaka Medical College

2-7 Daigakuchou, Takatsukishi

Osaka 569-8686

Japan

Fax: +81-52-7635233

oguratakeshi0411@yahoo.co.jp 